\title{
Exploring MeV Gamma Rays from Dark Matter Annihilation and Evaporating Primordial Black Holes in the GRAMS Experiment
}

\author{
Jonathan LeyVa ${ }^{a, *}$ on behalf of the GRAMS Collaboration \\ (a complete list of authors can be found at the end of the proceedings) \\ ${ }^{a}$ Northeastern University, Department of Physics \\ 360 Huntington Ave, Boston, MA 02115, USA \\ E-mail: j.leyva@northeastern.edu
}

The upcoming GRAMS (Gamma-Ray and AntiMatter Survey) experiment aims to provide unprecedented sensitivity to a poorly explored region of the cosmic gamma-ray spectrum from 0.1-100 MeV, often referred to as the "MeV gap". Utilizing Liquid Argon Time Projection Chamber (LArTPC) technology to detect these MeV gamma rays, GRAMS has the potential to uncover crucial details behind a variety of processes in multi-messenger astrophysics. Various theories on particle interactions beyond the standard model predict that dark matter annihilations may contribute to the cosmic gamma spectrum via monochromatic gamma emissions (spectral lines), the annihilation of decay products, and the radiation of electromagnetically charged final states (FSR). MeV gamma rays may also be emitted from primordial black holes (PBHs) that are currently gaining interest as candidates for dark matter. By looking for the Hawking radiation from such objects, GRAMS can likely probe for ultra-light PBHs, which theoretically may comprise the majority of dark matter seen in the Universe. Here, we will describe how the analyses of the targeted gamma-ray regime will enable GRAMS to uniquely and complementarily place constraints on low-mass dark matter models.

$37^{\text {th }}$ International Cosmic Ray Conference (ICRC 2021)

July 12th - 23rd, 2021

Online - Berlin, Germany 


\section{Introductions}

Close to a century ago, in the early 1930s, a milestone was reached in our understanding of galaxies that would radically change our perspective on the composition of the Universe. Utilizing the Doppler effect to analyze the motion of stars in our home galaxy, Jan Oort was among the first astronomers to notice a discrepancy in the rotational dynamics of the Milky Way [1]. Applying Newtonian mechanics to analyze these motions, it was concluded that the detectable luminous matter represented only a fraction of what should truly be present in our galaxy. Despite Oort regarding this discrepancy as a failure to detect dimmer celestial objects, the following decades of improved theory and instrumentation has only confirmed the reality of this "missing mass". By modern estimations, the entirety of detectable baryonic matter accounts for a paltry $\sim 5 \%$ of the Universe's mass-energy content. It is believed that $\sim 72 \%$ of the mass-energy density of the Universe can be accounted for by dark energy, the driving force behind the accelerating expansion of spacetime [2] [3] .The last 22-23\% is known as dark matter and is the missing mass that has troubled astronomers and physicists for the last several decades.

Assuming the validity of Newtonian dynamics and general relativity, it is not unreasonable to consider dark matter (DM) being comprised of a new, undiscovered class of particle that has yet to be added to the standard model. So far, DM remains discernible exclusively via gravitational interaction. Several theories have been devised to solve the DM mystery without the addition of an unknown particle (such as MOND, modified Newtonian dynamics [4]), yet these often struggle to explain certain phenomena, like those arising from the galactic merger 1E 0657-56, better known as the Bullet Cluster [5].

Considering DM as a particle, several methods of detection are popular today. Some experiments aim to detect dark matter particles via direct interactions with the lattice or atoms in the volume of their detector, i.e. nuclear or electron recoil. SuperCDMS [6] [7] and LUX-ZEPLIN [8] are examples of direct searches that employ different target materials and detection mechanisms. In the world of collider experiments, complimentary searches have been done with the hope that elusive dark matter particles are seen in the aftermath of high energy particle collisions. For this work, however, we will focus on the indirect search for DM via its annihilation or decay into detectable standard model particles such as photons, light leptons, and mesons at $\mathrm{MeV}$ energies.

As more and more parameter space is excluded for DM being made of WIMPs (Weakly Interacting Massive Particles, in the GeV-TeV mass range), there is building interest in probing the $\mathrm{MeV}$ domain for any signatures that may be left by a lighter DM candidate. Several successful MeV missions like NuSTAR [9] and Fermi-LAT [10] have thus far explored energies up to 10-100 keV (hard X-ray band) and above $20 \mathrm{MeV}$ (high-energy gamma rays) respectively. This leaves a widely unexplored region famously referred to as the "MeV gap" from $\sim 0.1-100 \mathrm{MeV}$. GRAMS and other upcoming $\mathrm{MeV}$ astronomy experiments will aim to close this gap with next-generation precision and better our understanding of many mysterious cosmological processes.

\section{GRAMS Detector}

The GRAMS detector is classified as a Liquid Argon Time Projection Chamber (LArTPC) and is expected to be sensitive to both antimatter particles at sub-GeV $(0.1-0.25 \mathrm{GeV} / \mathrm{n})$ energy scales as 


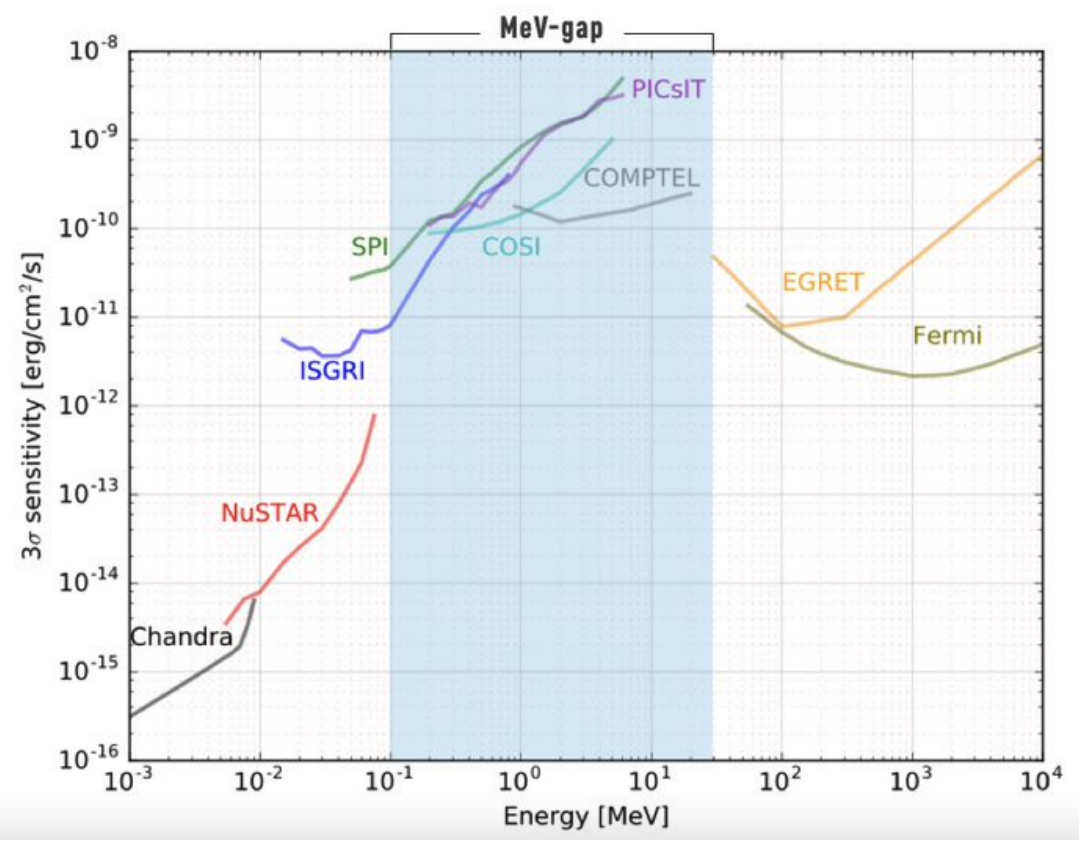

Figure 1: Current status of $\mathrm{MeV}$ exploration

well as $\mathrm{MeV}$ gamma rays. For the detection of gamma rays, the LArTPC at the heart of the GRAMS detector is used as a Compton camera and as a particle tracking calorimeter [11]. Such a detector has the potential to probe many cosmic mysteries that surround objects like pulsars, blazars, black holes, neutron star mergers, etc. For this work, we will focus on the ability of GRAMS to explore the $\mathrm{MeV}$ spectrum that may result from DM self-annihilation or decay as well as the evaporation of primordial black holes [12].

\section{Gamma Rays from DM Annihilation}

In the context of indirect DM searches, it is theorized that DM particles of a certain mass range may self-annihilate directly into gamma rays or into other standard model particles that can annihilate or decay further into gamma rays [13]. The entire annihilation spectrum is summarized by the following expression, with each term accounting for one of three types of contribution to the overall spectrum.

$$
\left.\frac{d N}{d E_{\gamma}}\right|_{\bar{\chi} \chi}=\left.\frac{d N}{d E_{\gamma}}\right|_{\bar{\chi} \chi, \text { line }}+\left.\frac{d N}{d E_{\gamma}}\right|_{\bar{\chi} \chi, \mathrm{dec} .}+\left.\frac{d N}{d E_{\gamma}}\right|_{\bar{\chi} \chi, \mathrm{FSR}}
$$

This spectrum can be computed for a variety of different models with the help of a free Python toolkit called Hazma [14]. In the first term, the annihilation or decay of DM directly into photons is considered. These are monochromatic emissions and therefore appear as spectral lines. The other two terms consider DM annihilating into leptonic or mesonic states, which in turn, contribute to the overall gamma ray spectrum via decay or final state radiation (FSR). This type of radiation includes but is not limited to Bremmstrahlung and synchrotron radiation. Using the annihilation spectrum 
in conjunction with parameters like the thermally averaged DM annihilation cross section $\langle\sigma v\rangle_{\chi \chi}$, dark matter mass $\left(m_{x}\right)$, and $\mathbf{J}$-factor, the total gamma-ray flux from DM annihilation per solid angle in a region of sky can be expressed as,

$$
\left.\frac{d \Phi}{d E_{\gamma}}\right|_{\bar{\chi} \chi}(E)=\left.\frac{\Delta \Omega}{4 \pi} \cdot \frac{\langle\sigma v\rangle_{\bar{\chi} \chi}}{2 f_{\chi} m_{\chi}^{2}} \cdot J \cdot \frac{d N}{d E_{\gamma}}\right|_{\bar{\chi} \chi}(E) .
$$

If DM is taken to be a Majorana, $f_{x}=1$ and if DM is assumed to have an antimatter partner, $f_{x}=2$. For this work, we only consider the case in which $f_{x}=2$. An expression called the J-factor is used in Eq. 2 to account for the expected abundance of DM in the region of interest and is a function of the angle subtended in the sky and the line-of-sight (LOS) distance to the target. For this work, a Navarro-Frenk-White (NFW) profile will be assumed for decaying or annihilating DM in our galaxy [15] [16]. Furthermore, a $5^{\circ} \mathrm{x} 5^{\circ}$ window pointed towards the galactic center will be considered for all following projections. The exponent in Eq. 3 is taken to be $\alpha=1$ for DM decay and $\alpha=2$ for DM annihilation, the latter to account for annihilating DM particle pairs.

$$
J \equiv \frac{1}{\Delta \Omega} \int_{\Delta \Omega} d \Omega \int_{\operatorname{LOS}} d l \rho(r(l, \psi))^{\alpha}
$$

For simplicity, a branching fraction of one hundred percent was considered while computing the differential gamma ray spectrum for each annihilation channel as seen in Fig. 2. Using the Hazma software toolkit, this spectrum can be computed for any annihilation channel by providing the free parameters of DM particle mass and an energy range for the produced photons.
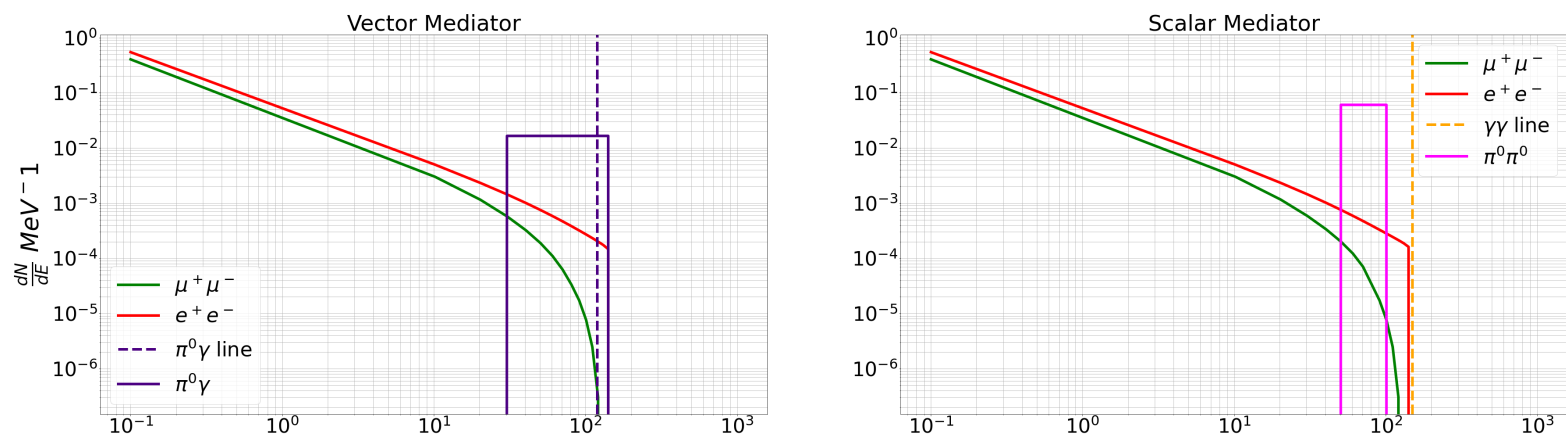

Figure 2: Annihilation Spectrum from kinematically allowed channels, 100\% Br. Fraction, computed by Hazma

\subsection{Sensitivity to DM Annihilation}

The same background model is used for all projections and includes contributions from atmospheric scattering as well as the diffuse, extragalactic background. The effective area functions for 
the GRAMS balloon and satellite missions can be seen in Fig. 3 alongside comparable missions like e-ASTROGAM [17] and AMEGO [18]. Close to $10 \mathrm{MeV}$, a cusp can be seen marking the transition between the Compton scattering and pair production regimes [11].
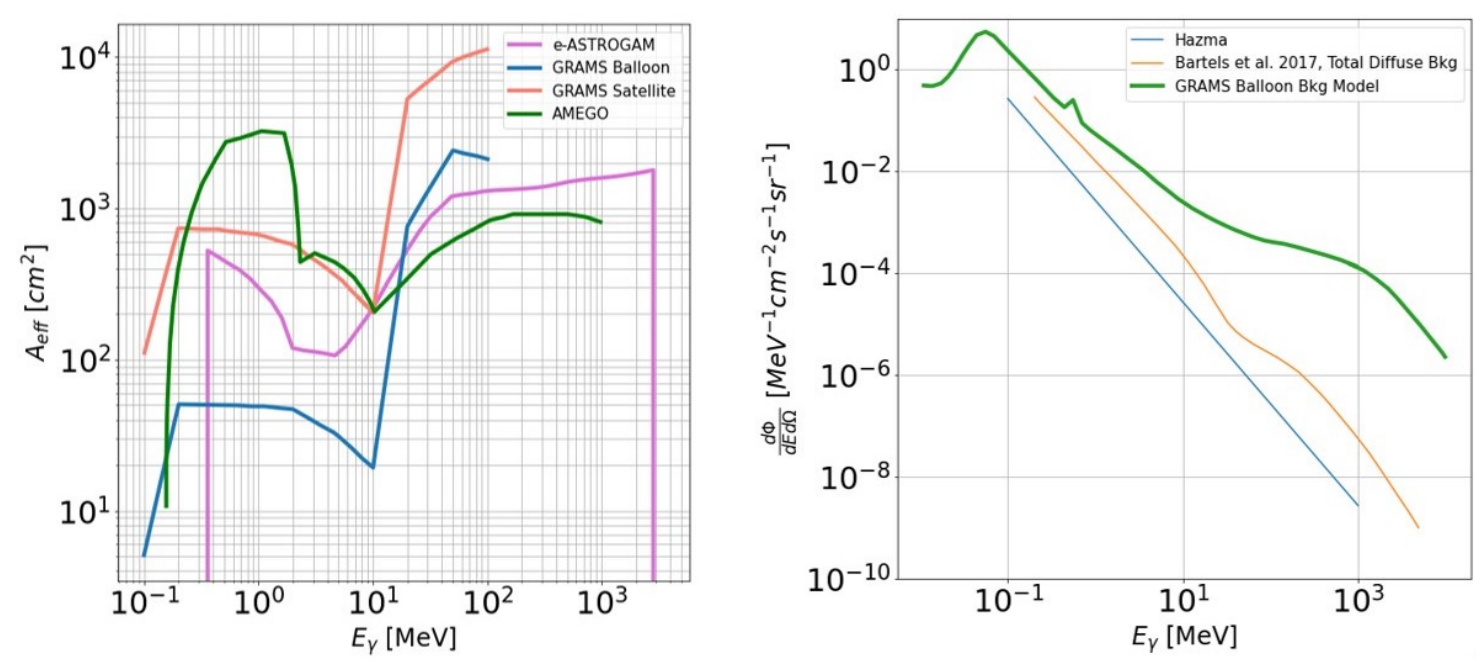

Figure 3: Left: Effective area functions of both GRAMS missions and comparable missions Right: GRAMS balloon background model was used for this work

Projections for GRAMS's sensitivity to the smallest, detectable $\langle\sigma v\rangle_{\chi \chi}$ for a given DM mass were done by considering Poissonian statistics for the signal-to-noise ratio, requiring a confidence level of $95 \%$ and an observation time of one year.
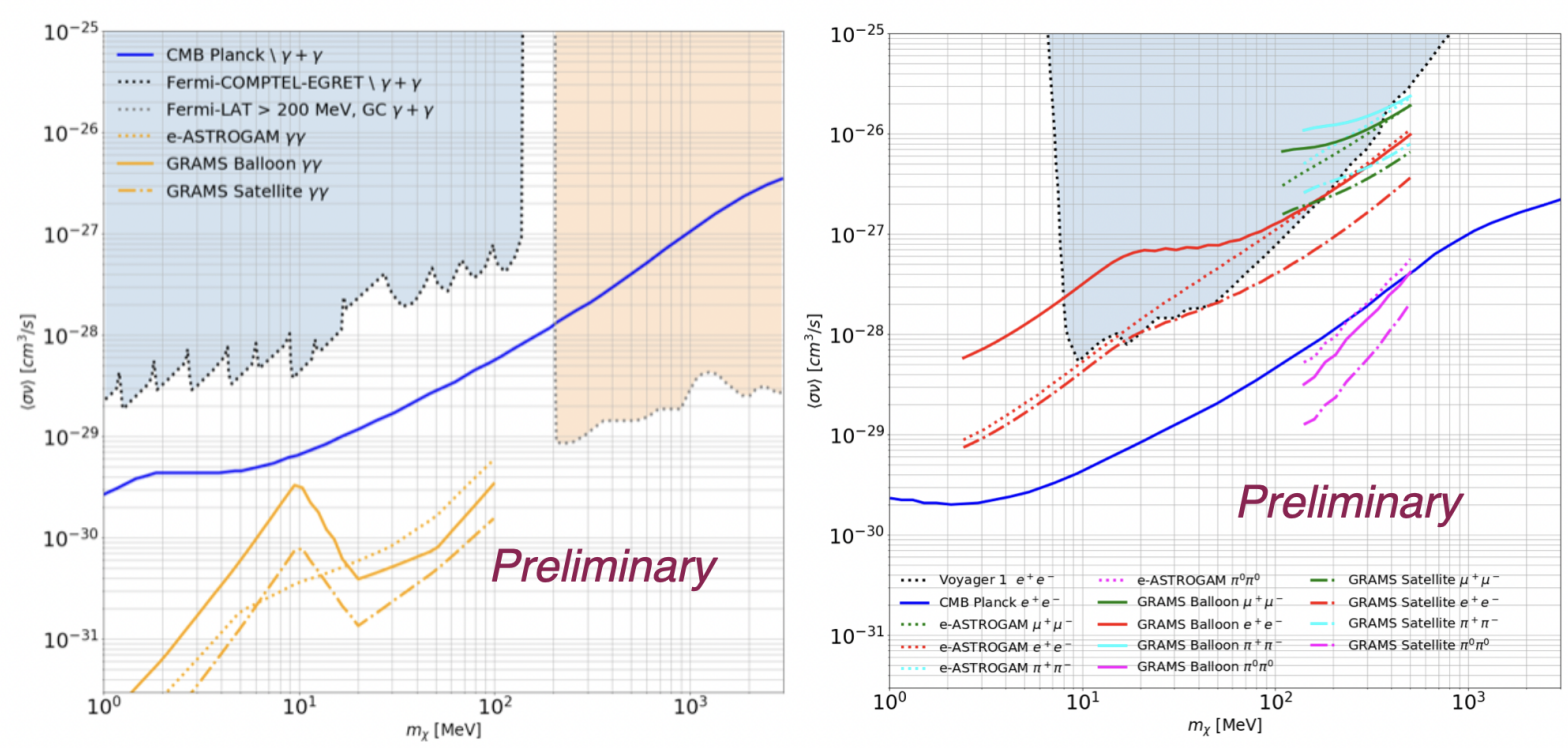

Figure 4: Left: Projected sensitivity to $\gamma \gamma$ annihilation channel, 95\% CL. Right: Projected sensitivity to $\mathrm{e}^{+}$ $\mathrm{e}^{-}$annihilation channel, 95\% CL.

Based on preliminary projections (Figure 4), the GRAMS detector will have the potential to probe unexplored parameter space in the hunt for particle DM. 


\section{PBH Evaporation}

Primordial black holes are thought to have formed in the very early Universe from extremely energy dense regions that existed shortly after the Big Bang. For some time, PBH's have been a topic of interest as candidates for DM, as they are non-baryonic, stable, and can plausibly explain results seen in microlensing data from observations of the Large Magellanic Cloud by the MACHO collaboration in the 1990s [19], [20]. These cosmically ancient black holes are relatively small in size for a $\mathrm{BH}$ and therefore evaporate quickly through intense, short wavelength Hawking radiation. However, PBH's at masses above $\sim 10^{14} \mathrm{~g}$ are expected to live longer than the age of the Universe and therefore should be present today [21].

Recently analyzed data from SPI onboard the INTEGRAL satellite have placed conservative constraints on PBH masses up to $\sim 10^{17} \mathrm{~g}$ that exclude them from comprising all of DM [12]. Furthermore, microlensing results from surveys of the Andromeda galaxy by the Subaru telescope have constrained PBH's above the mass of $\sim 10^{23} \mathrm{~g}$ from making up the majority of dark matter [22], [23]. Although a large area of parameter space has been ruled out, there still remains an unexplored PBH mass range that could plausibly account for all DM.

Under the assumption that the density profile of PBH's follows that of DM in the Milky Way, we employ the same J-factor expression that is used to model decaying DM, i.e. Eq. 3 where $\alpha=1$

Using the same aforementioned observation time, ROI, effective area functions, and background model, projections were made to estimate the sensitivity of upcoming MeV missions to PBH's with masses that fall within this unexplored parameter space. To compute the differential flux emitted by PBH's, their Hawking evaporation gamma-ray spectrum was computed with the help of an open source code called BlackHawk [24].
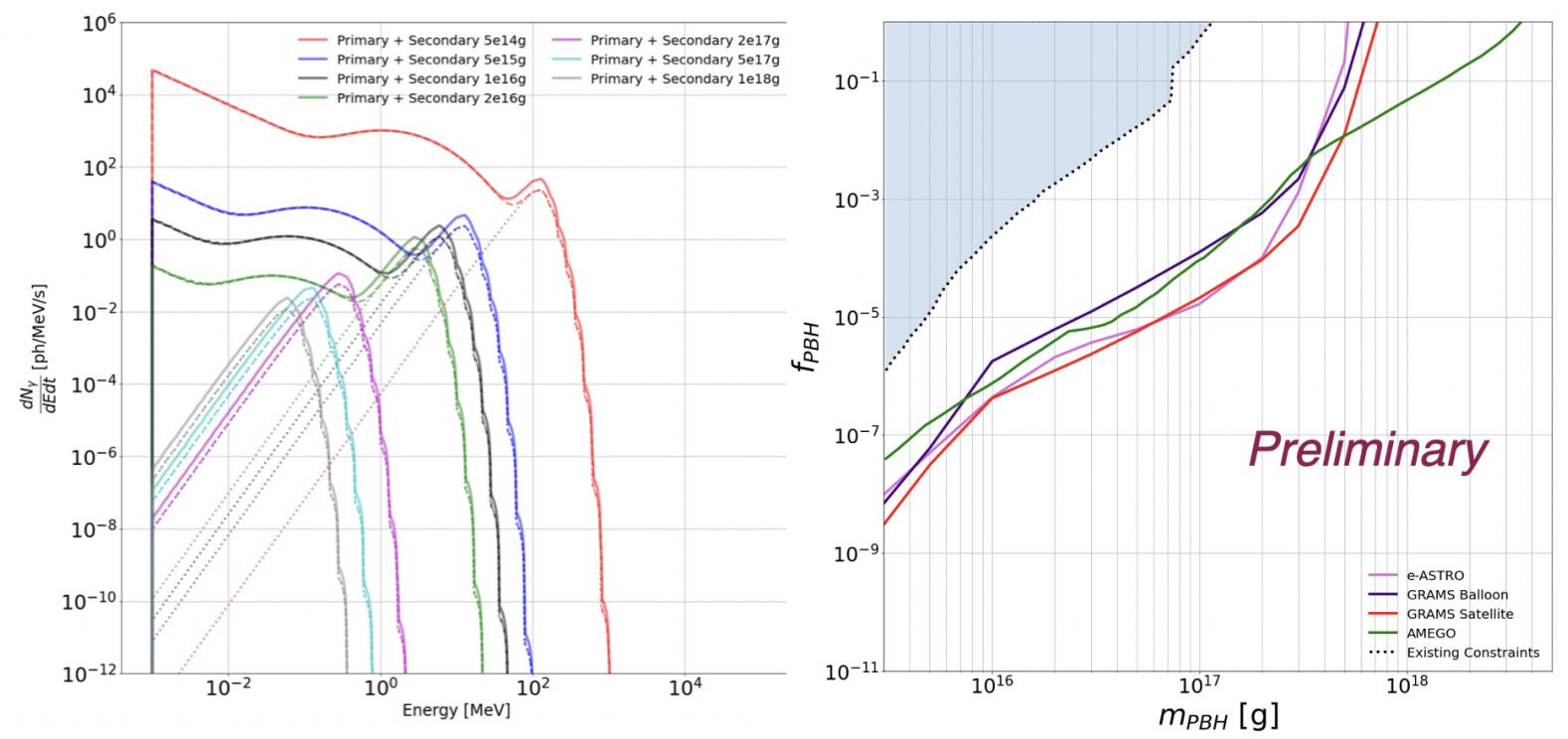

Figure 5: Left: Hawking evaporation spectrum computed by BlackHawk for a single PBH, several masses plotted. Right: Existing and projected constraints on fraction of PBH's comprising DM, 95\% CL

Along with several other upcoming MeV missions, GRAMS can explore new parameter space for PBHs that may constitute some or all of DM (Figure 5). Particularly, possible signals emanating 
from PBH's with masses that are not yet excluded for comprising all of DM, can be investigated.

\section{Conclusions}

GRAMS is a next-generation project that can perform an indirect DM search through antimatterbased particle detection as well as $\mathrm{MeV}$ gamma ray observations. The planned balloon flight in 5 years can extensively explore the $\mathrm{MeV}$ gap with an order of magnitude improved sensitivity compared to previous missions. Moreover, it is expected that the satellite mission improves sensitivity by 1-2 orders of magnitude over the balloon mission. Both GRAMS missions (especially satellite) have promising potential to push bounds on phase space for decaying and annihilating DM as well as constraints on PBH abundance.

\section{Acknowledgments}

This work was supported by Tsuguo Aramaki's start-up funds from Northeastern University. We acknowledge support from JSPS KAKENHI 610 grant numbers 20K22355 and 20H00153. We also acknowledge support from Barnard College and Columbia University.

\section{References}

[1] J. H. Oort, The force exerted by the stellar system in the direction perpendicular to the galactic plane and some related problems, Bulletin of the Astronomical Institutes of the Netherlands 6 (1932) 249.

[2] E. Komatsu, J. Dunkley, M. Nolta, C. Bennett, B. Gold, G. Hinshaw, N. Jarosik, D. Larson, M. Limon, L. Page, et al., Five-year wilkinson microwave anisotropy probe* observations: cosmological interpretation, The Astrophysical Journal Supplement Series 180 (2009) 330.

[3] P. A. Ade, N. Aghanim, M. Arnaud, M. Ashdown, J. Aumont, C. Baccigalupi, A. Banday, R. Barreiro, J. Bartlett, N. Bartolo, et al., Planck 2015 results-xiii. cosmological parameters, Astronomy \& Astrophysics 594 (2016) A13.

[4] M. Milgrom, A modification of the newtonian dynamics as a possible alternative to the hidden mass hypothesis, The Astrophysical Journal 270 (1983) 365-370.

[5] R. Massey, J. Rhodes, R. Ellis, N. Scoville, A. Leauthaud, A. Finoguenov, P. Capak, D. Bacon, H. Aussel, J.-P. Kneib, et al., Dark matter maps reveal cosmic scaffolding, Nature 445 (2007) 286-290.

[6] R. Agnese, A. Anderson, M. Asai, D. Balakishiyeva, R. B. Thakur, D. Bauer, J. Billard, A. Borgland, M. Bowles, D. Brandt, et al., Search for low-mass weakly interacting massive particles using voltage-assisted calorimetric ionization detection in the supercdms experiment, Physical review letters 112 (2014) 041302.

[7] R. Agnese, A. J. Anderson, M. Asai, D. Balakishiyeva, R. B. Thakur, D. Bauer, J. Beaty, J. Billard, A. Borgland, M. Bowles, et al., Search for low-mass weakly interacting massive particles with supercdms, Physical review letters 112 (2014) 241302.

[8] D. Akerib, C. Akerlof, D. Y. Akimov, A. Alquahtani, S. Alsum, T. Anderson, N. Angelides, H. Araújo, A. Arbuckle, J. Armstrong, et al., The lux-zeplin (lz) experiment, Nuclear Instruments and Methods in Physics Research Section A: Accelerators, Spectrometers, Detectors and Associated Equipment 953 (2020) 163047.

[9] F. A. Harrison, W. W. Craig, F. E. Christensen, C. J. Hailey, W. W. Zhang, S. E. Boggs, D. Stern, W. R. Cook, K. Forster, P. Giommi, et al., The nuclear spectroscopic telescope array (nustar) high-energy x-ray mission, The Astrophysical Journal 770 (2013) 103.

[10] W. Atwood, A. A. Abdo, M. Ackermann, W. Althouse, B. Anderson, M. Axelsson, L. Baldini, J. Ballet, D. Band, G. Barbiellini, et al., The large area telescope on the fermi gamma-ray space telescope mission, The Astrophysical Journal 697 (2009) 1071.

[11] T. Aramaki, P. O. H. Adrian, G. Karagiorgi, H. Odaka, Dual mev gamma-ray and dark matter observatory-grams project, Astroparticle Physics 114 (2020) 107-114. 
[12] R. Laha, J. B. Muñoz, T. R. Slatyer, I n t e g r a 1 constraints on primordial black holes and particle dark matter, Physical Review D 101 (2020) 123514.

[13] R. Bartels, D. Gaggero, C. Weniger, Prospects for indirect dark matter searches with mev photons, Journal of Cosmology and Astroparticle Physics 2017 (2017) 001.

[14] A. Coogan, L. Morrison, S. Profumo, Hazma: a python toolkit for studying indirect detection of sub-gev dark matter, Journal of Cosmology and Astroparticle Physics 2020 (2020) 056.

[15] J. F. Navarro, C. S. Frenk, S. D. White, A universal density profile from hierarchical clustering, The Astrophysical Journal 490 (1997) 493.

[16] M. Cirelli, G. Corcella, A. Hektor, G. Hütsi, M. Kadastik, P. Panci, M. Raidal, F. Sala, A. Strumia, Pppc 4 dm id: a poor particle physicist cookbook for dark matter indirect detection, Journal of Cosmology and Astroparticle Physics 2011 (2011) 051.

[17] A. De Angelis, V. Tatischeff, I. A. Grenier, J. McEnery, M. Mallamaci, M. Tavani, U. Oberlack, L. Hanlon, R. Walter, A. Argan, et al., Science with e-astrogam: A space mission for mev-gev gamma-ray astrophysics, Journal of High Energy Astrophysics 19 (2018) 1-106.

[18] J. McEnery, J. A. Barrio, I. Agudo, M. Ajello, J.-M. Álvarez, S. Ansoldi, S. Anton, N. Auricchio, J. B. Stephen, L. Baldini, et al., All-sky medium energy gamma-ray observatory: exploring the extreme multimessenger universe, arXiv preprint arXiv:1907.07558 (2019).

[19] C. Alcock, R. Allsman, D. Alves, T. Axelrod, A. Becker, D. Bennett, K. Cook, K. Freeman, K. Griest, J. Guern, et al., The macho project large magellanic cloud microlensing results from the first two years and the nature of the galactic dark halo, The Astrophysical Journal 486 (1997) 697.

[20] A. M. Green, B. J. Kavanagh, Primordial black holes as a dark matter candidate, Journal of Physics G: Nuclear and Particle Physics 48 (2021) 043001.

[21] A. Coogan, L. Morrison, S. Profumo, Direct detection of hawking radiation from asteroid-mass primordial black holes, Physical Review Letters 126 (2021) 171101.

[22] H. Niikura, M. Takada, N. Yasuda, R. H. Lupton, T. Sumi, S. More, T. Kurita, S. Sugiyama, A. More, M. Oguri, et al., Microlensing constraints on primordial black holes with subaru/hsc andromeda observations, Nature Astronomy 3 (2019) 524-534.

[23] N. Smyth, S. Profumo, S. English, T. Jeltema, K. McKinnon, P. Guhathakurta, Updated constraints on asteroid-mass primordial black holes as dark matter, Physical Review D 101 (2020) 063005.

[24] A. Arbey, J. Auffinger, Blackhawk: A public code for calculating the hawking evaporation spectra of any black hole distribution, The European Physical Journal C 79 (2019) 1-26. 


\section{Full Authors List: GRAMS Collaboration}

K. Aoyama ${ }^{1}$, T. Aramaki ${ }^{2}$, J. Asaadi ${ }^{3}$, L. Fabris ${ }^{4}$, Y. Ichinohe ${ }^{5}$, Y. Inoue ${ }^{6,7,8}$, G. Karagiorgi ${ }^{9}$, D. Khamgulyan ${ }^{10}$, M. Kimura ${ }^{1}$, J. Leyva ${ }^{2}$, R. Mukherjee ${ }^{11}$, T. Nakasone ${ }^{1}$, H. Odaka ${ }^{7,12,13}$, K. Perez ${ }^{14}$, M. Sakurai ${ }^{1}$, W. Seligman ${ }^{9}$, S.

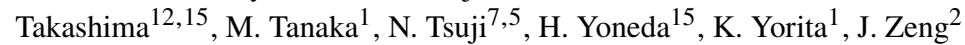

${ }^{1}$ Waseda University, 3-4-1, Okubo, Shinjuku, Tokyo 169-8555, Japan. ${ }^{2}$ Northeastern University, 360 Huntington Ave, Boston, MA 02115, USA. ${ }^{3}$ University of Texas-Arlington, Arlington, TX 76019, USA. ${ }^{4}$ Oak Ridge National Laboratory, Oak Ridge, TN 37830, USA. ${ }^{5}$ Department of Physics, Rikkyo University, 3-34-1 Nishi Ikebukuro, Toshima, Tokyo 171-8501, Japan. ${ }^{6}$ Department of Earth and Space Science, Graduate School of Science, Osaka University, Toyonaka, Osaka 560-0043, Japan. ${ }^{7}$ Interdisciplinary Theoretical \& Mathematical Science Program (iTHEMS), RIKEN, 2-1 Hirosawa, Wako, Saitama 351-0198, Japan. ${ }^{8}$ Kavli Institute for the Physics and Mathematics of the Universe (WPI), The University of Tokyo, Kashiwa 277-8583, Japan. ${ }^{9}$ Columbia University, 538 West 120th Street, New York, NY 10027, USA. ${ }^{10}$ Graduate School of Artificial Intelligence and Science, Rikkyo University, 3-34-1 Nishi Ikebukuro, Toshima, Tokyo 171-8501, Japan. ${ }^{11}$ Barnard College, 3009 Broadway, New York, NY 10027, USA. ${ }^{12}$ Department of Physics, The University of Tokyo, 7-3-1 Hongo, Bunkyo, Tokyo 113-0033, Japan. ${ }^{13}$ Research Center for the Early Universe, The University of Tokyo, 7-3-1 Hongo, Bunkyo, Tokyo 113-0033, Japan ${ }^{14}$ Massachusetts Institute of Technology, 77 Massachusetts Ave, Cambridge, MA 02139, USA. ${ }^{15}$ RIKEN Nishina Center, 2-1 Hirosawa, Wako, Saitama 351-0198, Japan. 